Krylov subspace iterative techniques: on the detection of brain activity with electrical impedance tomography

Polydorides, N. and Lionheart, W.R.B. and McCann, H. 2002

MIMS EPrint: 2006.240

Manchester Institute for Mathematical Sciences

School of Mathematics

The University of Manchester

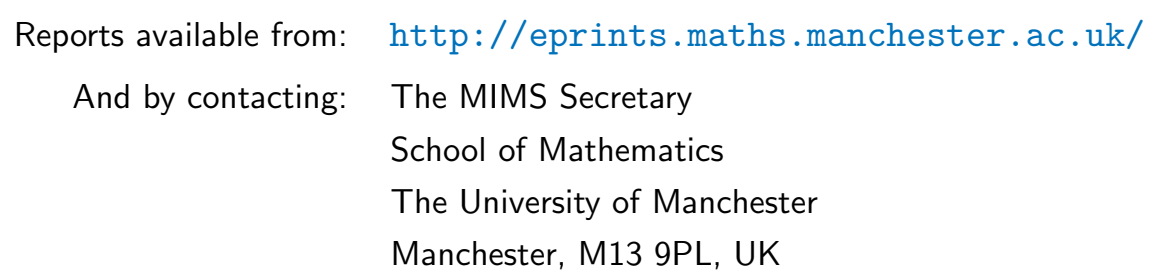

ISSN 1749-9097 


\title{
Krylov Subspace Iterative Techniques: On the Detection of Brain Activity With Electrical Impedance Tomography
}

\author{
Nick Polydorides, William R. B. Lionheart, and Hugh McCann*
}

\begin{abstract}
In this paper, we review some numerical techniques based on the linear Krylov subspace iteration that can be used for the efficient calculation of the forward and the inverse electrical impedance tomography problems. Exploring their computational advantages in solving large-scale systems of equations, we specifically address their implementation in reconstructing localized impedance changes occurring within the human brain. If the conductivity of the head tissues is assumed to be real, the preconditioned conjugate gradients (PCGs) algorithm can be used to calculate efficiently the approximate forward solution to a given error tolerance. The performance and the regularizing properties of the PCG iteration for solving ill-conditioned systems of equations (PCGNs) is then explored, and a suitable preconditioning matrix is suggested in order to enhance its convergence rate. For image reconstruction, the nonlinear inverse problem is considered. Based on the Gauss-Newton method for solving nonlinear problems we have developed two algorithms that implement the PCGN iteration to calculate the linear step solution. Using an anatomically detailed model of the human head and a specific scalp electrode arrangement, images of a simulated impedance change inside brain's white matter have been reconstructed.
\end{abstract}

Index Terms-Brain activity, computational efficiency, conjugate gradients, electrical impedance tomography, regularization.

\section{INTRODUCTION}

$\mathbf{I}^{\prime}$ N ELECTRICAL impedance tomography (EIT) a number of low-frequency current patterns are injected from the boundaries of a conductive volume by some electrodes while a number of linearly independent voltage measurements are captured on others. The imaging capabilities of EIT are based on the fact that the knowledge of an adequate set of boundary measurements along with an accurate model of the volume and some prior information can be used to reconstruct the electrical conductivity distribution in the interior of the volume at the time when the measurements were captured.

One of the most challenging projects involving EIT is the detection of brain activity under some physiological phenomena that are known to cause local and temporal conductivity changes within the human brain tissue [3]. Classical examples are the

Manuscript received August 28, 2001; revised February 22, 2002. Asterisk indicates corresponding author.

N. Polydorides is with the Department of Electrical Engineering and Electronics, UMIST, M60 1QD Manchester, U.K.

W. R. B. Lionheart is with the Department of Mathematics, UMIST, M60 1QD Manchester, U.K.

*H. McCann is with the Department of Electrical Engineering and Electronics, UMIST, P.O. Box 88, M60 1QD Manchester, U.K.

Publisher Item Identifier 10.1109/TMI.2002.800607. visual and auditory stimuli [21] as well as several ambulatory cases like migraines, strokes and epilepsy [4], [14], [19]. Despite the fact that the effects of these conditions vary in their magnitude and duration, each one tends to affect a particular area of the brain. This paper is primarily focused on the brain response to visual stimulation seeking to explore how this can be accurately and efficiently recovered in the prospect of a robust and reliable on-line monitoring based on EIT. In [15], Holder et $a l$. have reported that the conductivity changes caused by this form of stimulus lie in the range $2.7 \%-4.5 \%$, and as such these are small enough to allow the consideration of a linear approximation to the nonlinear inverse conductivity problem.

For a conductive volume of fixed boundaries and a certain conductivity distribution, the forward problem requires the calculation of the potential distribution inside the volume when known current patterns are injected from its boundaries. The mathematical modeling of the forward problem incorporates an elliptic partial differential equation derived from Maxwell's equations in the low-frequency range and some mixed boundary conditions [8]. The problem is often solved numerically rather than analytically using finite-element approximations, which necessitate a finite-element model of geometrical and structural characteristics similar to those of the real volume. Neglecting any magnetization effects, when the volume is a linear and isotropic medium $\Omega \subset \mathbb{R}^{3}$ with boundary $\partial \Omega$, the physical system is governed by the following set of equations also known as the complete electrode model (CEM) [25]. If $\tau$ is a point in the volume, then

$$
\begin{aligned}
\nabla \cdot(\sigma(\tau) \nabla u(\tau)) & =0, \quad \tau \in \Omega \\
u(\tau)+z_{l} \sigma(\tau) \frac{\partial u(\tau)}{\partial \nu} & =U_{l}, \quad \tau \in e_{l} \\
\int_{e_{l}} \sigma(\tau) \frac{\partial u(\tau)}{\partial \nu} d s & =I_{l}, \quad \tau \in e_{l} \\
\sigma(\tau) \frac{\partial u(\tau)}{\partial \nu} & =0, \quad \tau \in \Gamma_{1} .
\end{aligned}
$$

In the above equations, $u$ is the potential distribution inside $\Omega$, $z_{l}$ is the contact impedance of the $l$ th electrode $e_{l}, U_{l}$ is the potential measured on $e_{l}$, and $I_{l}$ is the current injected by $e_{l}$ and $\nu$ the outward unit normal. For the same equations, $\Gamma_{2}$ denotes the surface underneath the electrodes, $\Gamma_{1}$ denotes the rest of the boundary, and $\sigma$ denotes the electrical conductivity which is taken as real and positive. Formulating the variational-Galerkin form of the forward problem as in [20] and adopting a finite- 
element method (FEM) approach, the forward problem can be expressed as a system of linear equations

$$
A u=b .
$$

In a system with $L$ electrodes attached at the boundaries of a mesh incorporating $n$ nodes and $k$ elements, if $\sigma \in \mathbb{R}^{k}$, then $A \in \mathbb{R}^{n+L \times n+L}$ is the sparse, symmetric and positive definite global conductance matrix, $u \in \mathbb{R}^{n+L}$ is the approximated potential distribution, and $b \in \mathbb{R}^{n+L}$ is the associated low-frequency current patterns. The existence and uniqueness of the solution are preserved by incorporating into the model some additional constraints regarding the applied currents and measured voltages. In effect, $\sum_{l=1}^{L} I_{l}=0$ and $\sum_{l=1}^{L} U_{l}=0$ are imposed [20]. The solution of the forward problem can be naively attempted using a conventional method like the Gaussian elimination with pivoting for instance, although usually more efficient techniques like the Cholesky method [22] are employed exploiting the symmetric structure of the matrix $A$.

\section{Conjugate Gradients (CGs)}

The CGs iteration as a technique for solving linear systems is based on the idea that a problem of a certain dimension can be projected into a lower dimension Krylov subspace [12]. In doing so, the original problem is effectively reduced to a sequence of lower dimension matrix problems. When applied to the system of equations (5) the solution obtained by the $n$th iteration will lie in the associated Krylov subspace generated by $A$ and $b$, like

$$
\begin{aligned}
u_{n} & \in \mathcal{K}_{n}(A ; b) \text { where } \\
\mathcal{K}_{n}(A ; b) & =\operatorname{span}\left\{b, A b, A^{2} b, A^{3} b, \ldots, A^{n-1} b\right\} .
\end{aligned}
$$

The algorithm originally derived from the Lanczos iteration [10], requires the coefficient matrix $A \in \mathbb{R}^{n \times n}$ to be symmetric and positive definite, thus, it can only be applied in EIT's forward computations when the conductivity $\sigma$ is real. Nevertheless, there are other Krylov subspace methods [2], [10] that are suitable for solving systems of equations where the coefficient matrix is likely to have complex or negative eigenvalues. Setting an initial estimate of the solution $u_{0}$, the CG algorithm applied to the system (5) can be described as

$$
\begin{aligned}
& r=b-A u_{0} \\
& \text { while }\left\|r_{n}\right\|_{2}>\gamma \text { do } \\
& n=n+1 \\
& \text { if } n=1 \\
& p=r \\
& \text { else } \\
& \beta_{n}=\left\|r_{n-1}\right\|_{2}^{2} /\left\|r_{n-2}\right\|_{2}^{2} \\
& p_{n}=r_{n-1}+\beta_{n} p_{n-1} \\
& \text { end } \\
& \alpha_{n}=\left\|r_{n-1}\right\|_{2}^{2} /\left(p_{n}^{T} A p_{n}\right) \\
& u_{n}=u_{n-1}+\alpha_{n} p_{n} \\
& r_{n}=r_{n-1}-\alpha_{n} A p_{n} \\
& \text { endwhile }
\end{aligned}
$$

where $\alpha_{n}$ is the step length, $r_{n}$ is the residual vector, $p_{n}$ is the search direction, and $u_{n}$ is the approximate solution at the $n$th iteration. If $\gamma$ is the error tolerance parameter, then the iterations progress until the condition $\left\|r_{n}\right\|_{2} \leq \gamma$ is satisfied. From the definition of $\alpha_{n}$, an important concept involved in this technique is the $A$-norm of a nonzero vector $p$, which according to [22] is defined as

$$
\|p\|_{A}=\sqrt{p^{T} A p}
$$

thus, $A$ must be square and positive definite. The fundamental principle behind the $\mathrm{CG}$ iteration is that instead of minimizing the two-norm of the residual $r_{n}$, the $A$-norm of the error function $\varepsilon_{n}=\tilde{u}-u_{n}$ is minimized, where $\tilde{u}$ is the exact solution of the forward problem such that $\tilde{u}=A^{-1} b$. In this sense, while the iterations are progressing, a unique sequence of iterates $u_{n} \in \mathcal{K}_{n}$ is generated with the property that at iteration $n$, the $A$-norm of the error $\varepsilon_{n}$ is minimized.

When dealing with real experimental measurements the accuracy of the forward calculations is often set by the precision of the measurement circuit, i.e., the error estimate in the actual measurements. As the system described in (5) is a discrete approximation, $u_{n}$ will be an approximate solution strongly depending on the quality and the smoothness of the finite-element model used. Setting appropriately the error tolerance parameter $\gamma$, the CG algorithm enables the calculation of the solution based on "how accurately" one aims to solve the system avoiding any unnecessary refinement computations. From the description of the algorithm, one can notice that the solution update $u_{n}$ is generated by moving a distance $\alpha_{n}$ in the current search direction. In addition, throughout the iterations the search directions $p_{n}$ and residual vectors $r_{n}$ maintain certain properties. More specifically, after $n$ iterations the residual vectors form the orthogonal basis $\left\{r_{0}, r_{1}, \ldots, r_{n}\right\}^{\perp}$ and the search directions are found to be mutually "A-orthogonal."

\section{Conjugate Gradients PReconditioning}

The efficiency of the CG algorithm when applied to linear systems is critically dependent on the eigenvalues of the coefficient matrix. In the optimum case, these will be clustered around a fixed positive number and, therefore, a few iterations will be adequate for the algorithm to reach convergence. The concept of preconditioning is based on the idea that one can drastically improve the properties of the coefficient matrix before the beginning of the iterations and consequently eliminate the computation time required. For an appropriately selected preconditioner $\Theta$, the forward problem described in (5) has a solution that is identical to the one of the system

$$
\Theta^{-1} A u=\Theta^{-1} b
$$

only in this case the convergence of the algorithm will depend on the properties of $\Theta^{-1} A$ rather than those of $A$ alone. A left preconditioner $\Theta$ is regarded suitable when it is positive definite and the factor $\Theta^{-1} A$ satisfies $\Theta^{-1} A \approx \gamma I$, condition $\left(\Theta^{-1} A\right)$ $<$ condition $(A)$ where $I$ is the identity matrix. A quite popular and efficient preconditioning option is the one arising from the incomplete Cholesky factorization of $A$ [10]. If $H$ is the upper triangular factor of $A$, then a preconditioner $\Theta$ is formed such as $\Theta=H^{T} H$ preserving the existence of a unique symmetric 


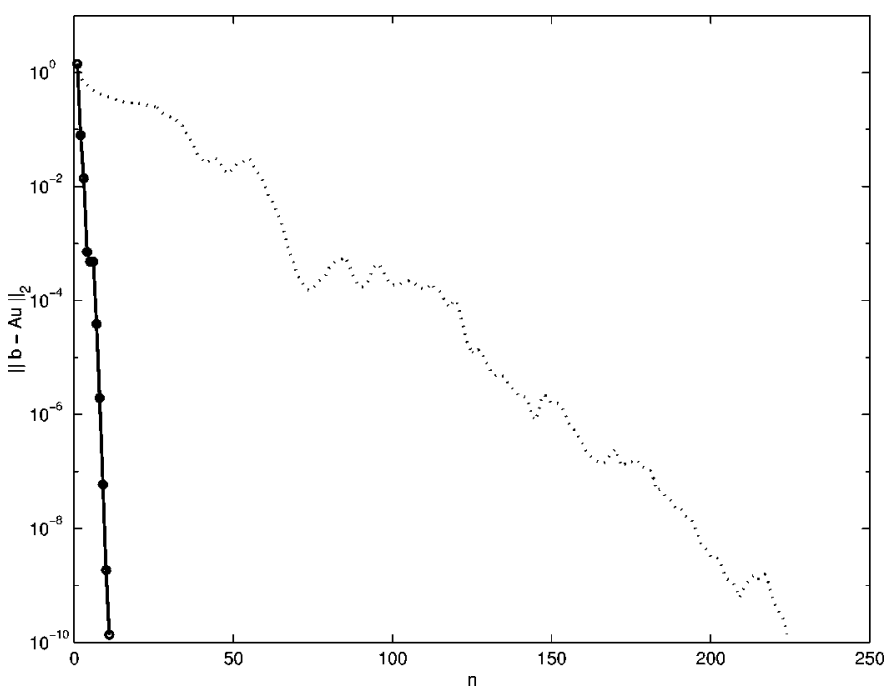

Fig. 1. A graph of the two-norm of the error residual $\{b-A u\}$ against the number of iterations. The graph shows a performance comparison between the CG algorithm with preconditioning (solid line) and without preconditioning (dotted line) when solving the forward problem to an error tolerance of $1.0 \times 10^{-10}$

positive definite matrix $G$ such that $\Theta=G^{2}$. Therefore, the system can now be formulated as

$$
\bar{A} \bar{u}=\bar{b}
$$

where $\bar{A}=G^{-1} A G^{-1}, \bar{u}=G u$ and $\bar{b}=G^{-1} b$. In Fig. 1, a performance comparison between the simple $\mathrm{CG}$ and the preconditioned CG algorithm (PCG) is shown when these are employed to solve the same forward problem. The graphs address explicitly the importance of preconditioning as the PCG algorithm manages to reach the desired error tolerance at a radically higher convergence rate.

\section{CG APPlied to NoRmal AND NeAR SiNGULAR EQUATIONS}

For the inverse problem, an approximation to the conductivity distribution in the interior of the volume has to be recovered from a finite set of boundary measurements. If a volume with initial conductivity distribution $\sigma_{0}$ is subjected to a set of current patterns $I$, then an array of boundary voltage measurements $U$ can be calculated according to the specific arrangement of the electrodes and the data collection protocol used. When a local perturbation in the conductivity $x$ occurs, the same current patterns will produce another array of boundary measurements such that the perturbation in the measurements $\delta U$ is always different from zero. The relationship between the perturbations in the interior conductivity distribution and the perturbations in the measurements of potential at the boundaries is nonlinear, however, a simple first approach to the problem is by formulating its linearization and then regularize in order to derive a stable solution. If in the continuous region $J$ is the Fréchet derivative of $U$ with respect to $\sigma$ for the nonlinear mapping $\sigma \mapsto U(\sigma)$, then in the discrete case the linearized forward problem takes the form of

$$
J x=\delta U
$$

where $x \in \mathbb{R}^{k}$ is the required perturbation in conductivity distribution that maps uniquely to the array of differential voltage measurements $\delta U \in \mathbb{R}^{m}$, and $J \in \mathbb{R}^{m \times k}$ the discrete linear forward operator or Jacobian matrix. As the system is ill-conditioned, $x$ cannot be calculated directly from (10), thus, even in the case where a $J^{-1}$ exists this would still be unbounded causing the solution $x$ to not depend continuously on the data $\delta U$. For this kind of problems, the conventional approach is to adopt some form of regularization in order to obtain a stable and unique solution. Usually this comes in the form of a weighting matrix that encompasses some prior information about the desired solution. One of the most widely used regularization techniques is the one based on Tikhonov's minimization scheme [8] and it is formulated as a least-squares problem with an extra side constraint. This improves the conditioning of the Jacobian by filtering a cluster of its smaller singular values, effectively preventing measurement noise from being amplified in the solution. If $x$ is small enough, a solution to the linearized inverse problem [7] can be obtained from the Tikhonov problem which is formulated as

$$
\arg \min \left\{\|J x-\delta U\|_{2}^{2}+\lambda\|\mathcal{D} x\|_{2}^{2}\right\}
$$

where $\mathcal{D}$ is the regularization matrix and $\lambda$ the strictly positive regularization parameter. If the interior conductivity distribution is known to be smooth, then $\mathcal{D}$ can be the discrete form of a differential operator.

Formulating the normal equations for the system (10) as

$$
J^{T} J x=J^{T} \delta U
$$

a CG oriented solution to the inverse problem can be evaluated by solving the minimization problem

$$
\arg \min \|J x-\delta U\|_{2}^{2} \text { s.t. } x \in \mathcal{K}_{n}\left(J^{T} J ; J^{T} \delta U\right)
$$

where $\mathcal{K}_{n}$ is now the $n$th Krylov subspace generated from the near singular coefficient matrix $J^{T} J$ and the right-hand-side vector $J^{T} \delta U$. The iterations (CGN) are quite similar to the standard CG iterations described in Section II, only this time the coefficient matrix is dense and the solution lies in a different subspace. A nonlinear solution can be recovered by employing the CGN iterations to estimate the linearized step solution within the Gauss-Newton algorithm such as

$$
\begin{gathered}
\sigma_{i+1}=\sigma_{i}+x_{i} \\
x_{i} \longleftarrow \mathcal{K}_{i}\left(J_{i}^{T} J_{i} ; J_{i}^{T} \delta U_{i}\right)
\end{gathered}
$$

where $\sigma_{i}$ is the $i$ th estimate of the solution, $J_{i}$ is the Jacobian calculated based on the $\sigma_{i}, \delta U_{i}=U-U\left(\sigma_{i}\right)$ is the $i$ th right-hand-side vector, $U$ the boundary measurements and $U\left(\sigma_{i}\right)$ the calculated measurements based on the $\sigma_{i}$. Apart from the efficient calculation of the linearized inverse solution, one should also consider the advances in the computation of the Jacobian matrix in each nonlinear iteration in (14). Using the PCG algorithm as in (9) to obtain the forward solutions required for the calculation of $J_{i}$ will effectively improve the efficiency of 
the whole nonlinear algorithm. Using an adjoint problem formulation [5], [7], the $(i+1)$ update of the Jacobian can be calculated as

$$
J_{i+1}(k, s)=-\int_{\Omega} \nabla u_{i}^{k} \cdot \nabla u_{i}^{s} d x d y d z
$$

where $u_{i}^{k}, u_{i}^{s}$ are the field solutions calculated based on the $i$ th conductivity distribution update and the $k$ th and $s$ th current patterns, respectively. It must also be quoted that techniques like the adjoint source method [1] can help forming $J_{i}^{T} \delta U$ factors very efficiently.

The intrinsic regularizing properties of the CGN algorithm [2], [12] can be easily verified. Although in principle the iterations target to minimize the $J^{T} J$-norm of the error function, in effect they cause the two-norm of the residual $r_{n}$ to be minimized. If $r_{n}=J x-\delta U$, then modifying (7) and solving for the error shows that after $n$ iterations the condition $\left\|r_{n}\right\|_{2} \rightarrow$ minimum is satisfied. In this case, if $\tilde{x}$ is the actual inverse solution and $\epsilon_{n}$ is the error norm such that $\epsilon_{n}=\tilde{x}-x_{n}$, then as the iterations progress the function $\left\|\epsilon_{n}\right\|_{J^{T} J}$ can be shown to be minimized

$$
\begin{aligned}
\left\|\epsilon_{n}\right\|_{J^{T} J}^{2} & =\epsilon_{n}^{T} J^{T} J \epsilon_{n} \\
& =\left(\tilde{x}-x_{n}\right)^{T} J^{T} J\left(\tilde{x}-x_{n}\right) \\
& =x_{n}^{T} J^{T} J x_{n}-2 x_{n}^{T} J^{T} J \tilde{x}+\tilde{x}^{T} J^{T} J \tilde{x} \\
& =x_{n}^{T} J^{T} J x_{n}-2 x_{n}^{T} J^{T} \delta U+\tilde{x}^{T} J^{T} \delta U \\
& =2 f\left(x_{n}\right)+\text { constant } \\
\text { where } f(x) & =\frac{1}{2} x^{T}\left(J^{T} J\right) x-x^{T} J^{T} \delta U .
\end{aligned}
$$

From the above, one may conclude that after $n$ iterations

$$
\exists x_{n} \cdot f\left(x_{n}\right) \longrightarrow \text { minimum over } \mathcal{K}_{n}\left(J^{T} J ; J^{T} \delta U\right) .
$$

Some simple algebra reveals that $f(x)$ can also be expressed as

$$
f(x)=\frac{1}{2}\|J x-\delta U\|_{2}^{2}
$$

from which it follows that the $n$th iterate minimizes $\left\|r_{n}\right\|_{2}^{2}$ over the same subspace. In addition, since

$$
\nabla f(x)=J^{T} J x-J^{T} \delta U
$$

the value of $x$ for which $f(x)$ is minimum is also an approximate solution to the ill-conditioned system (12) [10].

\section{IMPLEMENTATION ISSUES}

Preconditioning properly the CGN iterations can cause dramatic improvements to their computational efficiency in the sense that a lower condition number will enable a higher convergence rate. For this reason, the diagonal matrix $R=$ $\operatorname{diag}\left(J^{T} J\right)$ was selected, which despite having quite small singular values its condition number is radically smaller compared to the one of the $J^{T} J$ matrix as these are maintained

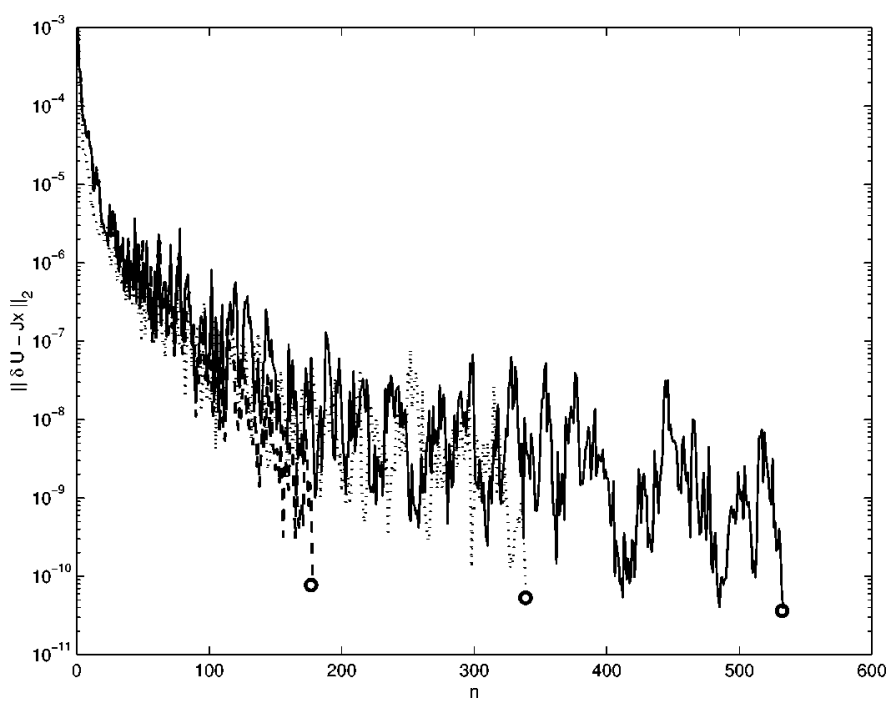

Fig. 2. The graph represents the norm of the error as a function of the number of iterations for the CGN algorithm applied to the near singular system (12) (solid line), the PCGN algorithm on the same system (dotted line) and finally the PCGN algorithm applied to the regularized system (19) (dashed line). The values of $\lambda$ and $\gamma$ used are $1.2 \times 10^{-12}$ and $6.8 \times 10^{-11}$, respectively.

well clustered. As it is shown in the graphs presented in Fig. 2 this selection enhances the performance of the algorithm by reducing the number of iterations required for convergence by almost a third.

Instead of applying the PCGs for normal equations (PCGN) algorithm to the system (12) directly, one may consider an alternative approach which is based on the PCGN iteration but it is even more efficient. Given the ill-conditioning of the system (12) one can improve its condition number before the iterations commence, effectively making their regularization task somewhat easier. The iterations can, therefore, be applied to a Tikhonov regularized system, and if the solution is assumed to be smooth a discrete approximation to the first differential operator $\mathcal{D}$ can be used modifying the system of normal equations as [11]

$$
\left(J^{T} J+\lambda \mathcal{D}^{T} \mathcal{D}\right) x=J^{T} \delta U
$$

In effect, a nonlinear inverse solution can be obtained by employing the Gauss-Newton algorithm incorporating the regularized PCGN iteration to compute the linear step solution as

$$
\begin{gathered}
\sigma_{i+1}=\sigma_{i}+x_{i} \\
x_{i} \longleftarrow \mathcal{K}_{i}\left(J_{i}^{T} J_{i}+\lambda \mathcal{D}^{T} \mathcal{D} ; J_{i}^{T} \delta U_{i}\right) .
\end{gathered}
$$

The graphs presented in Fig. 2 verify that the convergence rate of the PCGN algorithm is considerably lower when solving the system (12) rather than the regularized system (19) [13]. The criteria used for the selection of the regularization parameter $\lambda$, its relation with the error tolerance parameter $\gamma$ and its effect on the convergence of the algorithm are discussed in [11], [12]. The discrete first differential operator $\mathcal{D} \in \mathbb{R}^{k \times k}$ is set by a smoothing parameter $\omega$ which controls the degree of correlation between neighboring pixels in the image. For the smoothing process, we have treated as neighboring the tetrahedral elements which share at least one vertex. If the element $l$ shares one vertex 
with the elements $\left\{n_{1}, \ldots, n_{i}\right\}$, then the corresponding entries in the $\mathcal{D}$ matrix are

$$
\mathcal{D}_{\text {update }}=\left\{\begin{array}{l}
\mathcal{D}\left(l,\left\{n_{1}, \ldots, n_{i}\right\}\right)=-\omega^{-1} \\
\mathcal{D}(l, l)=\sum_{j=1}^{i} \mathcal{D}\left(l, n_{j}\right) .
\end{array}\right.
$$

Another alternative approach to the algorithm (14) is to modify the PCGN iteration in a way that the computational efficiency is not compromised by the size of the the normal equations coefficient. As it appears from the (12) and (19) the coefficient matrix $J^{T} J \in \mathbb{R}^{k \times k}$ where $k$ is the number of mesh elements, dominates the computational complexity of the system. To overcome these computational limitations a modified version of the PCGN algorithm [10] can be employed where by introducing the dummy variable $y \in \mathbb{R}^{m}$ the computationally expensive system (12) is transformed as

$$
\begin{aligned}
J x & =\delta U \\
J J^{T} y & =\delta U \\
x & =J^{T} y .
\end{aligned}
$$

In this case, the PCGN iterations are applied to the system (22b) solving for $y$ until the desired tolerance is reached. The value of $y$ at which the algorithm finally converges is then used to calculate the associated value of $x$ as in (22c). If $y_{0}$ is an initial guess for the solution of the system (22b) and $\Theta$ the preconditioning matrix such as $\Theta=\operatorname{diag}\left(J J^{T}\right)$, the modified PCGN iteration can be described as

$$
\begin{aligned}
& r_{0}^{y}=\delta U-J J^{T} y_{0} \\
& M=\Theta^{-1} \\
& \text { while }\left\|r_{n}\right\|_{2}>\gamma \text { do } \\
& n=n+1 \\
& z_{n}=M r_{n} \\
& \text { if } n=1 \\
& p=r \\
& \text { else } \\
& \beta_{n}=r_{n-1}^{T} z_{n-1} / r_{n-2}^{T} z_{n-2} \\
& p_{n}=z_{n-1}+\beta_{n} p_{n-1} \\
& \text { end } \\
& \alpha_{n}=r_{n-1}^{T} z_{n-1} /\left(p_{n}^{T} J J^{T} p_{n}\right) \\
& y_{n}=y_{n-1}+\alpha_{n} p_{n} \\
& r_{n}=r_{n-1}-\alpha_{n} J J^{T} p_{n} \\
& \text { endwhile } \\
& x_{n}=J^{T} y_{n} \\
& r^{x}=\delta U-J x
\end{aligned}
$$

where $\gamma$ is the error tolerance. From the above description, it is obvious that the value of $y_{n}$ that minimizes the residual $\| \delta U-$ $J J^{T} y_{n} \|_{2}^{2}$ also minimizes $\|\delta U-J x\|_{2}^{2}$ as the two residual vectors $r$ and $r^{x}$ can be easily proved to be equal. This modified version of the PCGN algorithm can also be used within the Gauss-Newton method to form an efficient nonlinear solver for the underdetermined problem such as

$$
\begin{aligned}
& \sigma_{i+1}=\sigma_{i}+x_{i} \\
& y_{i} \longleftarrow \mathcal{K}_{i}\left(J_{i} J_{i}^{T} ; \delta U_{i}\right) \\
& x_{i}=J_{i}^{T} y_{i} .
\end{aligned}
$$

TABLE I

The Conductivity Values Assigned to the Head Tissues IN $\mathbf{Z m}^{-1}$, ALONGSIDE THE NUMBER OF ELEMENTS ENCAPSULATED IN EACH OF THE MODEL TISSUES

\begin{tabular}{ccc}
\hline Tissue & Conductivity & No of elements \\
\hline scalp & 0.172 & 17584 \\
skull & 0.067 & 11981 \\
csf & 1.540 & 3359 \\
grey matter & 0.345 & 7347 \\
white matter & 0.150 & 4033 \\
\hline
\end{tabular}

In problems where bulky models are involved, the computational efficiency becomes a crucial issue for the reconstruction. The large number of mesh elements is often the reason that compromises the inverse computations on moderate machines, however, under certain conditions these can be radically simplified [1]. Consider for instance the situation where two distinct volumes $\Omega_{1} \subset \mathbb{R}^{3}$ and $\Omega_{2} \subset \mathbb{R}^{3}$ form the measurement volume $\Omega$ in a way that $\Omega=\Omega_{1} \cup \Omega_{2}$. If all the possible conductivity changes can be solely confined within say $\Omega_{1}$, one can truncate the domain of the discrete forward mapping (10) in a way that only the columns referring to the elements of $\Omega_{1}$ are included in the inverse computations. Restricting the range of the inverse problem by avoiding to calculate the solution where we are confident about its value maintains the Jacobian matrix within easily handled sizes and the time required for the computation of the solution within reasonable levels. In the anatomically detailed model employed in this study, the majority of the elements are allocated in tissues whose electrical properties are unlikely to change during the controlled conditions that we investigate. Tissues like the skin and the skull for instance, which encapsulate almost $67 \%$ of the overall number of elements, can be safely regarded as passive as these are expected to maintain their conductivity constant throughout the measurement acquisition process [16].

\section{Simulated RESUltS}

To perform the simulations we have used a three-dimensional (3-D) model of the human head constructed based on the information provided by the visible human project [26], comprising 9047 vertices connected in 44304 first-order tetrahedral elements. Although the skull and the white matter are known to be anisotropic [9], in this paper we regard all the tissues involved as linear isotropic media. The conductivity values assigned to them are summarized in Table I [18], [21]. The specific brain area targeted in this application is the visual cortex which is mainly consisted of the primary visual area and the visual association area. In this case, we have simulated a local conductivity increase of $4 \%$ within the occipital lobe, slices of which appear at the left side of the Fig. 4. This inhomogeneity can be considered to be equivalent to the effect of the visual stimulation on a heavily anaesthetized human subject [15].

A number of previous studies [17] have reported on the performance of single plane electrode systems in applications involving volumes of complicated geometrical and structural characteristics, emphasizing the effects caused by the absence of 3-D measurements on both the quality of the reconstructed image and the convergence of the reconstruction algorithms. 



Fig. 3. The 16 electrodes attached on the human head. Views of the arrangement from the front and the back.

The number and the positioning of the electrodes on the head's surface are among the issues where practical limitations are likely to arise. Although in principle one would aim to maximize their number, locating them throughout the surface of the scalp, a large number of electrodes and consequently a large number of measurements, will introduce delays in both the data acquisition cycle and the image reconstruction as the size of the problem will grow. In this concept it is also important to consider the relation between the data acquisition cycle and the "lifecycle" of the targeted effect. In real experiments, prolonged measurement cycles that are likely to occur in the presence of multiple current patterns, may distort the measurements because the magnitude of the particular effect is rather fast and periodic [16]. Unless time-varying models are adopted it is imperative that each measurements gathered refers to the same conductivity distribution, thus, the duration of the data acquisition cycle is a crucial factor if we are to consider the physiological effect static while the measurements are captured [24].

To meet the above specifications, a 3-D 16-electrode configuration was developed aiming to allow: 1) suitable current patterns to penetrate the resistive skull setting up an adequate field in the interior; 2) linearly independent multiplane measurements between closely located electrodes; and 3 ) the collection of the majority of the data from the boundary surface near the particular area of interest, i.e., the back area of the head. In order to pass an adequate electric current through the relatively high-resistive skull, among others some current patterns from diametrically located electrodes were also injected. In addition, care was taken to place some of these electrodes close to the eye sockets and ear holes, exploiting skull's structural characteristics. As far as the measurement patterns are concerned, most of the electrodes have been deliberately placed at the back area of the scalp close to where the the targeted effect was "expected" to occur, in order to enhance the system's sensitivity in that particular region. The exact positions of the 16 electrodes on the scalp are those indicated in Fig. 3. From this arrangement, a total of 19 current patterns were injected and 369 boundary voltage measurements were obtained by forward calculations. To perform the simulations in realistic conditions where the measurements are contaminated with a certain amount of noise, the measurements were infused with a Gaussian noise signal of zero mean and standard deviation of $10^{-5}$ of the norm of the measurements. This form of noise can be easily associated with the instrumentation noise introduced by the data acquisition circuit [6].

In order to be able to provide some form of assessment to the results obtained, one must consider what characteristics a successfully reconstructed image should possess, mainly in terms of its spatial resolution and its utility for medical diagnosis. Based on the particular simulated inhomogeneity, an acceptable image should indicate a single conductivity increase, symmetrically situated at the back side of the head, bounded within the brain matter tissue, and having geometrical characteristics similar to those of the simulated inhomogeneity. The images presented at the left column of Fig. 4 indicate the exact location of the simulated impedance change in slices deployed throughout the inhomogeneity's volume. The relevant slices from the 3-D nonlinear inverse solution, which corresponds to an error norm of $1.26 \times 10^{-4}$ are those appearing at the right column of the same figure. The images are extracted from the solution obtained by the third Gauss-Newton iteration using the PCGN algorithm to compute the linearized step as in (20). The reconstruction shows a localized impedance increase of magnitude similar to the one of the simulated change, situated at the back area of the brain matching the position where the original inhomogeneity has been simulated. Despite the presence of the resistive skull, the change has been successfully detected having most of its geometrical characteristics (symmetry and boundary shape) transferred into the image. In terms of its spatial resolution, the reconstructed change appears to fit reasonably well within the boundaries of the simulated one. As the system is heavily underdetermined the attempt to solve the problem with $k$ degrees of freedom using a radically smaller set of data $m$ will cause a systematic correlation among the elements of the solution that correspond to nearby pixels. As a result, when the solution is projected on to the pixels a smoothing effect is observed. The nonlinear inverse problem was attempted using both of the PCGN-based approaches described in Section V. At first, the Gauss-Newton PCGN algorithm was applied to the regularized system as in (20) using as preconditioner the $\operatorname{diag}\left(J^{T} J\right)$ matrix, while the problem was later solved using the underdetermined version of the PCGN algorithm (23) preconditioned with the diagonal $\operatorname{diag}\left(J J^{T}\right)$. In both cases, the linearized inverse problem was solved to an error tolerance of $10^{-8}$ for the iterative solution, and the two methods have accomplished similar performance with respect to their computational efficiency and the spatial resolution of the reconstructed images.

On a benchmark test based on the model of Table I, the PCGN algorithm as in (20) computed the linearized inverse solution after only $62 \%$ of the floating point operations per second (flops) required by the generalized Tikhonov solver (11), while the underdetermined version of the PCGN algorithm (23) reached convergence after $66 \%$ of the flops executed by the Tikhonov method. With regards to the forward computations, a similar test showed that the PCG algorithm (9) required only $56 \%$ of the flops executed by the Cholesky method when solving the forward problem to a tolerance of $1 \times 10^{-12}$. 


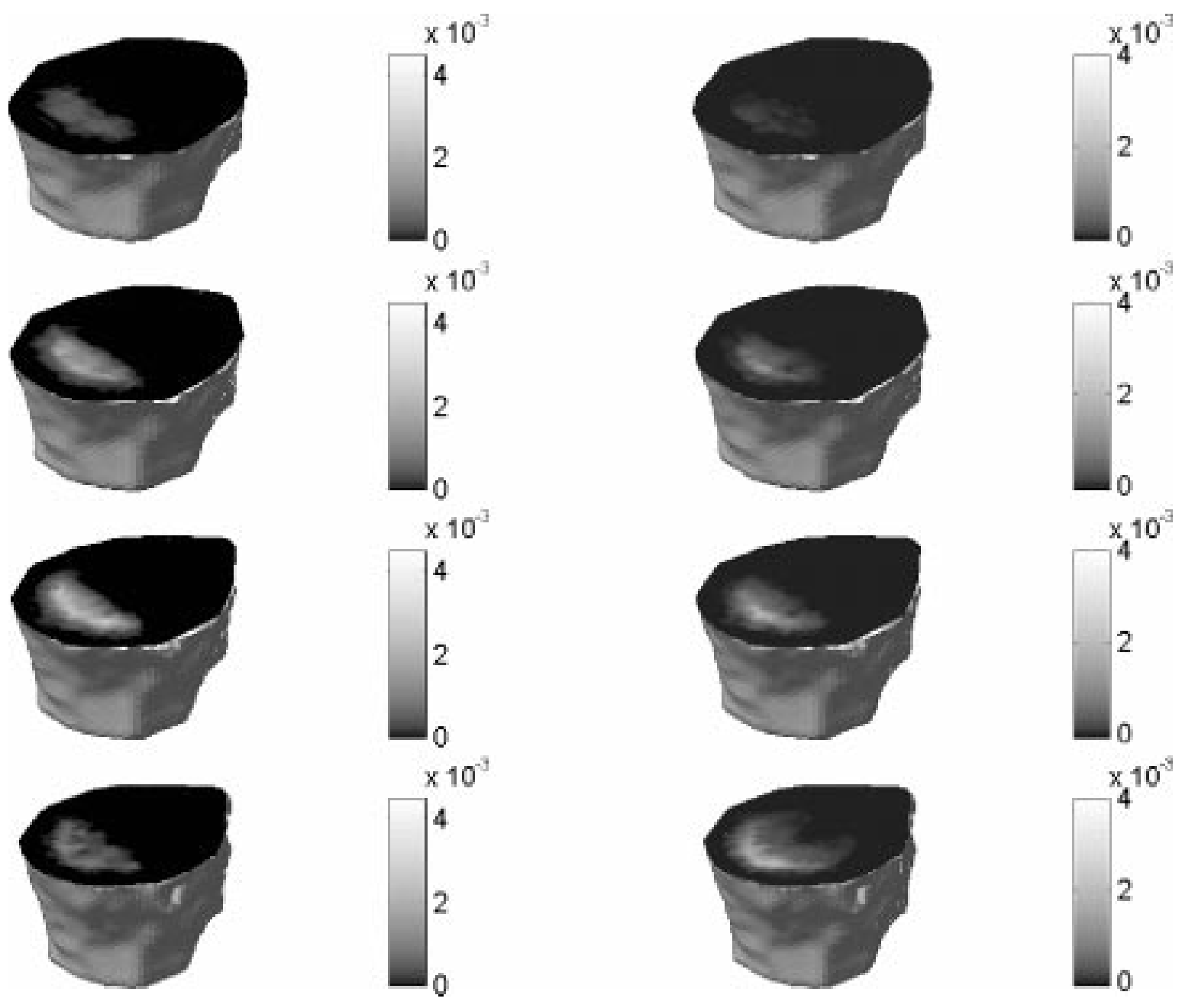

Fig. 4. On the left slices indicating the simulated inhomogeneity and on the right the corresponding slices from the reconstructed change in conductivity distribution. The images are extracted from the third Gauss-Newton iterative solution.

\section{CONCLUSION}

When the conductivity distribution is real the PCG iterations can drastically improve the efficiency of the forward computations. Solving the problem to the accuracy level required according to the error estimate in the actual measurements, the algorithm avoids to perform any unnecessary refinement computations. For the nonlinear inverse problem, two PCGN-based algorithms have been employed and preconditioned in order to calculate efficiently the linear step solution within the Gauss-Newton iterative method for solving nonlinear problems. Their performance in calculating the linearized inverse solution and the updated sensitivity matrix at each iteration demonstrates that even in the presence of bulky finite-element models, the nonlinear inverse conductivity problem can be solved efficiently and accurately. With regard to the specific biomedical application, the suggested 3-D scalp electrode arrangement was shown to achieve a local sensitivity enhancement in the specific area of interest.

\section{ACKNOWLEDGMENT}

The authors would like to acknowledge the help of the SCI Institute at the University of Utah and especially D. Weinstein for providing the human head models. They would also like to thank R. Kikinis of the Brigham and Women's Hospital, Boston, MA, and P. Krysl from the California Institute of Technology (CalTech), Pasadens, for their contribution in generating the meshes.

\section{REFERENCES}

[1] S. R. Arridge, "Topical review: Optical tomography in medical imaging," Inverse Prob., vol. 15, pp. R41-R93, 1999.

[2] R. R. Arridge and M. Schweiger, "The use of multiple data types in time-resolved optical absorption and scattering tomography (TOAST)," Proc. SPIE, vol. 2035, pp. 218-229, 1993.

[3] K. G. Boone, D. Barber, and B. Brown, "Review imaging with electricity: Report on the European concerted action on impedance tomography," J. Med. Eng. Technol., vol. 21, no. 6, pp. 201-232, 1997.

[4] K. Boone, A. M. Lewis, and D. S. Holder, "Imaging of cortical spreading depression by EIT: Implications for localization of epileptic foci," Physiol. Meas., vol. 15, no. Suppl 2a (6), pp. A189-98, 1994.

[5] W. R. Breckon, "Image Reconstruction in Electrical Impedance Tomography," Ph.D., Oxford Brookes Polytech., Oxford, U.K., 1990.

[6] B. H. Brown and A. D. Seagar, "Applied potential tomography-Data collection problems," presented at the Proc. IEE Int. Conf. Electric and Magnetic Fields in Medicine and Biology, London, U.K., 1985.

[7] M. Cheney, D. Isaacson, J. Newell, S. Simske, and J. Goble, "NOSER: An algorithm for solving the inverse conductivity problem," Int. J. Imag. Syst, Tech., vol. 2, 1990

[8] M. Cheney, D. Isaacson, and J. Newell, "Electrical impedance tomography," SIAM Rev., vol. 41, no. 1, pp. 85-101, 1999. 
[9] T. C. J. Facs, H. A. Van der Meij, J. C. De Munck, and R. M. Heethaar, "Topical review. The electrical resistivity of human tissues $(100 \mathrm{~Hz}-10$ MHz): A meta analysis of review studies," Physiol. Meas., vol. 20, pp. R1-R10, 1999.

[10] H. G. Golub and C. F. Van Loan, Matrix Computations, third ed: The Johns Hopkins University press, 1996.

[11] W. W. Hager, "Iterative methods for nearly singular linear systems," SIAM J. Scientific Computing, vol. 22, no. 2, pp. 747-766, 2000.

[12] M. Hanke, "Conjugate gradient type methods for ill-posed problems," in Pitman Research Notes in Mathematics. Harlow, Essex, U.K.: Longman House, 1995.

[13] P. C. Hansen, Rank Deficient Ill-Posed Problems. Philadelphia, PA: SIAM, 1998.

[14] D. S. Holder, C. A. Gonzalez-Correa, T. Tidswell, A. Gibson, G. Cusick, and R. H. Bayford, "Assessment and calibration of a low-frequency system for electrical impedance tomography (EIT), optimized for use in imaging brain function in ambulant human subjects," Proc. NY Acad. Sci., vol. 873, pp. 512-519, 1999.

[15] D. S. Holder, A. Rao, and Y. Hanquan, "Imaging of physiologically evoked responses by electrical impedace tomography with cordical electrodes in an anaesthesised rabbit," Physiol. Meas., vol. 17, pp. A179-A186, 1996.

[16] E. R. Kandel, J. H. Schwartz, and T. M. Jessell, Principles of Neural Science, 4th ed. New York: McGraw-Hill, 2000, ch. 25-27.

[17] W. R. B. Lionheart, "Boundary shape and electrical impedance tomography," Inverse Prob., vol. 14, no. 1, 1998.
[18] F. T. Oostendorp, J. Delbeke, and F. D. Stegeman, "The conductivity of the human skull: Results of in vivo and in vitro measurements," IEEE Trans. Biomed. Eng., vol. 47, Nov. 2000.

[19] R. J. Sadleir and R. A. Fox, "Detection and quantification of intraperitonial fluid using electrical impedance tomography," IEEE Trans. Biomed. Eng., vol. 48, Apr. 2001.

[20] E. Somersalo, M. Cheney, and D. Isaacson, "Existence and uniqueness for electrode models for electric current computed tomography," SIAM J. Appl. Math., vol. 52, 1992.

[21] C. M. Towers, H. McCann, M. Wang, P. C. Beatty, C. J. D. Pomfrett, and M. S. Beck, "3D simulation of EIT for monitoring impedance variations within the human head," Physiol. Meas., vol. 21, pp. 119-124, 2000.

[22] L. N. Trefethen and D. Bau III, Numerical Linear Algebra. Philadelphia, PA: SIAM, 1997.

[23] M. Vauhkonen, D. Vadasz, J. P. Kaipio, E. Somersalo, and P. A. Karjalainen, "Tikhonov regularization and prior information in electrical impedance tomography," IEEE Trans. Med. Imag., vol. 17, pp. 285-293, Apr. 1998.

[24] M. Vauhkonen, P. A. Karjalainen, and J. P. Kaipio, "Dynamical electrical impedance tomography," IEEE Trans. Biomed. Eng., vol. 45, pp. 486-493, Apr. 1998.

[25] P. Vauhkonen, M. Vauhkonen, T. Savolainen, and J. Kaipio, "Three-dimensional electrical impedance tomography based on the complete electrode model," IEEE Trans. Biomed. Eng., vol. 46, Sept. 1999.

[26] The Visible Human Project ${ }^{\circledR}$. National Library of Medicine, Bethesda MD. [Online]. Available: http://www.nlm.nih.gov 